\title{
Research on Pedestrian's Detection Based on the Integration of AdaBoost Algorithm and Shape Features
}

\author{
Ying Yang ${ }^{1, a}$, Yugang $\mathrm{Ma}^{2, b}$, Xiaodong Guo ${ }^{3, \mathrm{c}}$ and Kun Jiao ${ }^{4, \mathrm{~d}}$ \\ $1,2,3,{ }^{4}$ School of Mechanical Engineering and Automation, Northeastern University, Shenyang \\ 110819, China \\ b mayugang2010@sina.com
}

Keywords: AdaBoost algorithm, pedestrian detection, classifier, Edge detection

\begin{abstract}
In this paper, propose a pedestrian detection method that based on AdaBoost algorithm and pedestrian shape features integration. First according to the collected pedestrian true, false sample, selected the characteristics of the extended class Haar, adopt AdaBoost algorithm training get pedestrian classifier to split the initial candidate region of all pedestrians in the image. In this paper, propose an adaptive threshold weight update method, significantly reduced the number of the characteristics of strong classifier, optimize the classifier structure, reduce the complexity of the algorithm; meanwhile, the online update detector, improving the reliability of the detector. Pedestrian leg have strong vertical edge symmetry characteristic so that extracted the vertical edge detection in the initial candidate region, According to the symmetry determine the vertical axis of symmetry, combined with the morphological characteristics of pedestrians to determine the width and height characteristics of the pedestrian, to determine the pedestrian candidate region, Finally, put a further validation to the pedestrian candidate region.
\end{abstract}

\section{Introduction}

With China's rapid increase in car ownership, frequency of road traffic accidents, especially the traffic accidents caused by vehicle and pedestrian collision, is the main reason for pedestrian casualties. It makes the application of safety driver assistance systems in our country with greater urgency and relevance. Detection of the pedestrian in front of the vehicle is one the Indispensable functions of the safety driver assistance systems. It can effectively help the driver timely respond to the external environment in the urban to avoid pedestrian collisions. For a pedestrian detection system, it should have a good real-time, low false alarm rate, good environment adaptability. Currently, the commonly used pedestrian segmentation method is edge detection, is mainly to split the pedestrian in road. This paper presents a pedestrian candidate region segmentation method based on the integration of the shape characteristics of the pedestrian and AdaBoost algorithm, extract the possible position of the pedestrians in the image, so that provide input for the effective identification of pedestrian.[1,2]

\section{Improved AdaBoost algorithm}

Improved sample weights update method. FPR: the number of negative samples that be wrongly classified into positive samples with the ratio of the total number of negative samples, means the misclassification rate of negative samples; FNR: the number of positive samples that be wrongly classified into negative samples with the ratio of the total number of positive samples, means the misclassification rate of positive samples.[3]

In this article, you need to update the sample weights under the FPR's impact. In order to adjust the impact of FPR or FNR to the weight update process, we introduce the adjustment factor $\mathrm{k}$ to adjust or change the expansion of the sample weight subject to the FPR or FNR level. The weight update process will be extended to: 


$$
\begin{aligned}
& \omega_{t+1, i}=\omega_{t, i}\left(\beta_{t}^{1-\varepsilon_{t}}\right)^{(1-F P R)^{k}} . \\
& \omega_{t+1, i}=\omega_{t, i}\left(\beta_{t}^{1-\varepsilon_{t}}\right)^{(1-F N R)^{k}} .
\end{aligned}
$$

When the negative sample distribution is concentrated while the positive sample distribution is dispersed, it is suitable to use (1-FPR) as the index of the sample weight correction so that increase the attention algorithm paid to the positive samples; When the positive sample distribution is concentrated while the negative sample distribution is dispersed, it is suitable to use (1-FNR) as the index of the sample weight correction so that increase the attention algorithm paid to the negative samples. When the positive and negative samples are more evenly distributed, use (I-FPR) or (I-FNR) as the index to correct the value of FNR or FPR as needed.

In the experiment on the set of points we can see the adjustment factor $\mathrm{k}$ on the impact of the weight update method: When $\mathrm{k}$ is greater than 1 , it can accelerate the degree of correction of the weight update algorithm; when $\mathrm{k}$ is less than 1 , it can reduce the degree of correction of weight update algorithm.

Threshold Adaptive weight updates method. This extended sample weights update algorithm can reduce the FNR and effectively limit the increase of the overall sample error rate but it can not control the degree of FNR reduced.

Here are the threshold adaptive weight update algorithm steps:

(1) Initialization, the initial sample weight is 1 / N, N is total number of samples;

(2) if $F N R>f_{1}, \omega_{t+1, i}=\omega_{t, i}\left(\beta_{t}^{1-\varepsilon_{t}}\right)^{(1-F P R)^{k}}$;

(3) Elseif $\quad F P R>f_{2}, \omega_{t+1, i}=\omega_{t, i}\left(\beta_{t}^{1-\varepsilon_{t}}\right)^{(1-F N R)^{k}}$;

(4) Else $\omega_{t+1, i}=\omega_{t, i}\left(\beta_{t}^{1-\varepsilon_{t}}\right)$;

(5) Global normalization sample set, into the next round of training.

Note: $\mathrm{f} 1<\mathrm{f} 2, \mathrm{k}<\mathrm{l}$, with a large number of experimental data, take $\mathrm{f} 1=0.05, \mathrm{f} 2=0.3, \mathrm{k}$ from empirical value of $1 / 3$.

The simulation results show that the threshold adaptive sample weights update method can rapidly reduce the value of FNR in less rounds of training in the case of ensuring the overall error rate unchanged. The following will be the research for the characteristics of pedestrian detection.

\section{Calculation of the pedestrian sample Eigen values}

In the pedestrian detection, usually analysis sample set class at first in order to identify the target sample class. In this article, the analytical methods used are based on features rather than pixels. The knowledge that the objects require classified have are encoded to get the features. We use the class of Haar feature here; this rectangular feature is more sensitive to the edge and the line segments and the Eigen value of the class Haar is calculated very quickly.[10]

The number of sample Features. We add a line feature (4) to the features of the original four rectangular; the original line feature (3) is rotated 90 degrees to form a new line feature. As shown in Table 1. 
Table 1 Characteristics template conditions of $(\mathrm{s}, \mathrm{t})$

\begin{tabular}{llllll}
\hline & haar_x2 & haar_y2 & haar_x3 & haar_y3 & haar_x2_y2 \\
\hline $\begin{array}{l}\text { Characteristics } \\
\text { template }\end{array}$ & & & & & \\
\hline (s,t) conditions & $(1,2)$ & $(2,1)$ & $(1,3)$ & $(3,1)$ & $(2,2)$ \\
\hline
\end{tabular}

Table 1 lists the $(\mathrm{s}, \mathrm{t})$ conditions of different rectangle characteristics.

The total number of the five kinds of class Haar features of Characteristics template in all $\mathrm{m} \times$ $\mathrm{n}$ sub-windows is the sum of the number of the rectangle characteristics that meet the above $5(\mathrm{~s}, \mathrm{t})$ conditions. That is:

$\Omega=\Omega(1,2)+\Omega(2,1)+\Omega(1,3)+\Omega(3,1)+\Omega(2,2)$

In particular, as the characteristics templates 1 and 2,3 and 4 have rotational symmetry, it can be further simplified to:

$$
\Omega=2 \times \Omega(1,2)+2 \times \Omega(1,3)+\Omega(2,2) .
$$

Calculation of the sample Eigen values. Each class Haar feature is composed of 2 to 4 rectangles which are used to detect edges, linear features and diagonal features. The calculation of these features is the sum of the gray Integration of the rectangular area.

$$
\text { Feature }_{I}=\sum_{i \in I=\{1, \cdots, N\}} \omega_{i}^{*} \operatorname{Re} c \operatorname{Sum}\left(r_{i}\right)
$$

where: $\omega \mathrm{i} \in \mathrm{R}$ which is the right of rectangle, RecSum (ri) is the gray Integration of the area Surrounded by the rectangle ri, $\mathrm{N}$ is the number of rectangular included.

The calculation of the class Haar feature is achieved by integrating the image. The integral image $\tilde{I}$ of the gray image $\mathrm{I}$ is defined as:

$$
\tilde{I}(u, v)=\int_{x=0}^{u} \int_{y=0}^{v} I(x, y) d x d y
$$

Visible, the value of a point in the integral image is the sum of the gray of a rectangle area in the integral image, the opposite corner of this rectangle area is the origin and this point. With the integral image, you can find a way to quickly calculate each Haar features just like the way to lookup tables. [4]

\section{Improved classifier training}

AdaBoost algorithm is a classifier algorithm. Its basic idea is to stack up a large number of simple classifiers that have common ability by a certain method to form a strong classifier that has strong classification ability. Connect some strong classifiers in series to form a cascade classifier to detect the image. Each strong classifier is trained by AdaBoost algorithm [11]

We can know from the algorithm training process, if the classifiers extracted are right for some samples classification, the weight of these samples will reduce; if the classification is wrong, increase the weight of these samples. Thus the simple classifiers that later training extracted will strengthen the training to the wrong classified samples. Ultimately, form a strong classifier by weight the combination of all weak classifiers. The experimental results showed that when training times $\mathrm{T}=200$, the formed strong classifier can get a good test results. 


\section{Segmentation experiment of pedestrian initial candidate regions}

Acquisitions of pedestrian training samples. Training samples are divided into true sample and false sample, their size is scaled into a uniform $16 \times 32$ pixel size. Training samples are get through the method of manual calibration, part of the training sample images shown in Figure 5.1. This paper finally gets a total of 1000 pedestrian training sample images, of which 520 are real sample images, 480 sample images are false.

Each level of the cascade classifiers is a strong classifier which is obtained by the AdaBoost algorithm training. Set the threshold for each level when training that can let most pedestrian samples (such as $99.9 \%$ ) pass.

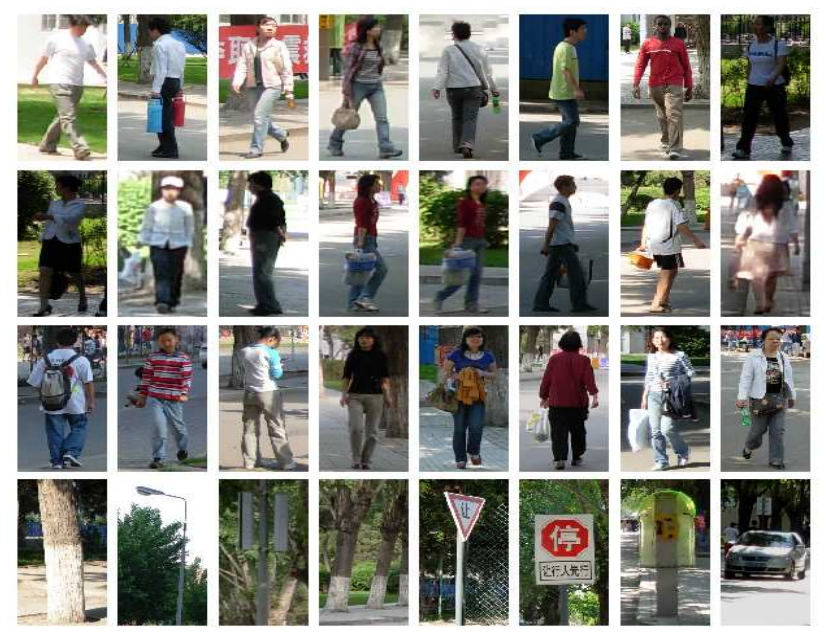

Fig. 1. Training samples in the image of the sample

When training strong classifier in each level, the main features should be used to constitute the strong classifiers that have simple structure. Locate these strong classifiers in the first few levels. The strong classifier should ensure a higher detection rate to the positive samples. But the fault detection rate of negative samples only required to within an acceptable range, with step by step strategy to reduce. [9]

Cascade classifier error detection rate and detection rate were:

Error detection rate:

$$
f=\sum_{i=1}^{n} H\left(x_{i}\right) \times\left(1-y_{i}\right) / \sum_{i=1}^{n}\left(1-y_{i}\right)
$$

Detection rate:

$$
d=\sum_{i=1}^{n} H\left(x_{i}\right) \times y_{i} / \sum_{i=1}^{n} y_{i} \text {. }
$$

As the second-class classification problem, so yi $=0$ or 1 . Clearly, in the cascade detector the smaller error rate is, the detection rate will be higher.

Assume cascade classifiers have $\mathrm{K}$ levels where the detection rate is di while error detection rate is fi, which $i=1,2 \ldots \mathrm{K}$. The final detection rate is $\mathrm{D}$, false detection rate is $\mathrm{F}$. Then the following formula holds:

False detection rate:

$$
F=\prod_{i=1}^{K} f_{i} \text {. }
$$

Detection rate:

$$
D=\prod_{i=1}^{K} d_{i} \text {. }
$$


Based on the above formula, as long as the expected rate of the final false detection rate and the detection rate is given, you can set the target value at each level of classifier. For the fi and di of each level, when $f_{1} \leq \sqrt[K]{F}$ and $d_{1} \leq \sqrt[K]{D}$, can meet the requirements.

In order to visually display the extracted Haar features, Figure 5.2 shows part of the class of Haar features' match to the pedestrian sample window which is included in the strong classifier obtained by training.

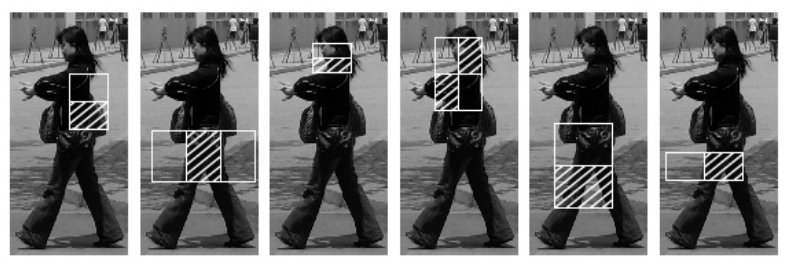

Fig. 2. Match of Pedestrians sample characteristics

Segmentation of pedestrian candidate region. Pedestrian segmentation, mainly refers to the cascade classifier finds the area that contains the object in the image and then display the area by a rectangular. In general, the classifiers use the different size of the window to scan several times and test the overlapping area every time. Combine these areas and return to the average rectangle in the big enough combination.[7]

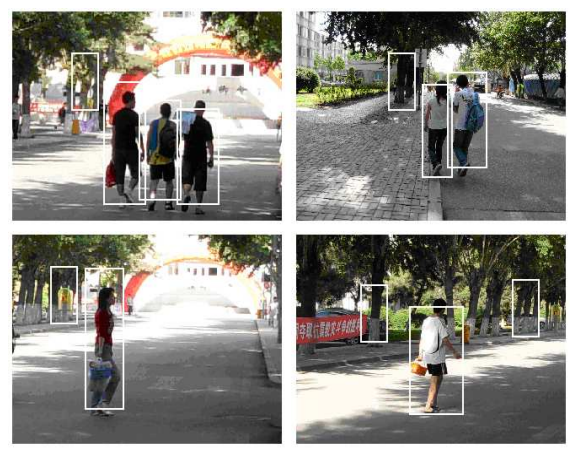

Fig. 3. Some pedestrians initial candidate regional division results

We change the size of the classifier rather than the image itself. Classifier is designed to be resized. The search window size is $32 \times 64$ pixels for pedestrian segmentation and the size of the image to be detected is $320 \times 240$. Experimental results show that, in order to detect pedestrians in various scales, two successive scans, enlarge the scale factor of search window 1.2 times each time is the best.

Cascade classifier training directly through the OpenCV library functions to achieve, and then load training by $\mathrm{C}++$ to get the classifier. Take real-time search for the CCD-collected image to get the line segmentation of the pedestrian candidate region. Figure 5.3 is part of the pedestrian segmentation results.

\section{Conclusions}

As the Information of visual sensor is rich, according to pedestrians shape characteristics and AdaBoost algorithm features, in this paper, we propose a pedestrian segmentation method which based on the Integration of pedestrians shape characteristics and AdaBoost algorithm. Using AdaBoost algorithm training get a pedestrian classifier with good recognition accuracy and use the resulting cascade classifier training obtain the initial candidate image in the pedestrian area. Secondly, propose an extended sample weight update method and a threshold adaptive weight 
update method. And the large number of simulation experiments find that the extended sample weight update can effectively limit the misclassification rate of positive samples in the case of the overall misclassification rate is certain. According to the actual needs, threshold adaptive weight update method can limit positive samples or negative samples misclassification rate within the expected range in the case of the overall misclassification rate is certain.

Reduce the complexity of the algorithm; the same time, online training can improve the scalability of the detector for the difficult samples; online update detector can improve the reliability of the detector. Timeliness is good, detect rapidly, the system detects a $320 \times 240$ size image only require $34 \mathrm{~ms}$. Due to actual traffic conditions, however, background on both sides of the road is complex, and thus brings a lot of difficulties and interference to the exact location for pedestrians also will be some of the false judgments, so it requires further confirmation to identify the candidate region that can achieve accurate identification of pedestrians.

\section{References}

[1]. M.Bertozzi,A.Broggi,P.Lombardi.Artificial Vision in Road Vehicles[J].Proceddings of the IEEE,July 2002, 90(7) :1258-1271.

[2]. M.Szarvas,A.Yoshizawa, M.Yamamoto and J.Ogata. Pedestrian Detection with Convolutional Neural Networks[C], Proc.IEEE Conference on Intelligent Transportation Systems, Tokyo,Japan,2005:224-229.

[3]. F.Xu and K.Fujimura.Pedestrian Detection and Tracking with Night Vision[J], IEEE Transactions Intelligent Transportation Systems, March 2005, 6(1): 63-71.

[4]. Massimo Bertozzi,Alberto Broggi.Pedestrian Detection for Driver Assistance Using Multiresolution Infrared Vision[J], IEEE Transcations on Vehicular Technology, Novermber 2004, 53(6):1666-1678.

[5]. M.Bertozzi, A.Broggi,M.Carletti,A.Fascioli, T.Graf, P.Grisleri, and M.Meinecke.IR pedestrian detection for advanced driver assistance systems[J], Lecture Notes Computer Science, 2003,2781:582-590.

[6]. D.M.Gavrila.Sensor-based pedestrian protection[J], IEEE Intelligent Systems, 2001, 16(6):77-81.

[7]. Wang Liang, Hu Weiming and Tan Tieniu, status identify based on tread[J], Computer Transaction, 2003, 26(3): 353-360.

[8]. Liang Wang,Huazhong Ning,Tieniu Tan and Weiming Hu.Fusion of Static and Dynamic Body Biometrics for Gait Recognition[J], IEEE Transcations on circuits and systems for Video Technology,February 2004,14(2):149-158.

[9]. Tian Guang, Qi Feihu and Zhu Wenjia, Walking people identify by single camera [J],System simulation transaction, 2006, 18(10): 2906-2910.

[10]. Dan Witzner Hansen, John Paulin Hansen, Mads Nielsen, Anders Sewerin, Johansen and Mikkel B.Stegmann. Eye Typing using Markov and Active Appearance Models. IEEE Workshop on Applications on Computer Vision, 2002, pp .132-136.

[11]. Kong Fanzhi, Zhang Xingzhou and Xie Yaoju, the technic of human face based on Adaboost[J], Application of science and technology, 2005, 32(6): 7-9.

[12]. Bertozzi M, Broggi A, Fascioli A, Graf T, Meinecke M M Pedestrian detection for driver assistance using multiresolution infrared vision. IEEE Transactions on Vehicular Technology, 2004, 53(6): 1666-1678. 